\title{
Massive pneumatosis intestinalis and free abdominal air: Is surgery always necessary?
}

\begin{abstract}
Pneumatosis intestinalis can have various causes, ranging from benign to life-threatening. In some cases diagnosis is followed by immediate surgery to rule out mesenteric ischemia. Here we report the case of a 19 year-old patient who was incidentally diagnosed with massive pneumatosis intestinalis and free abdominal air following norovirus infection after hematopoietic stem cell transplantation for acute myeloic leukemia. Although the patient was almost completely asymptomatic, diagnostic laparoscopy was performed to rule out perforation or ischemia. Intraoperatively, massive pneumatosis of the colon without any other pathological findings was visible, so no further intervention was necessary and the patient could be discharged from hospital 5 days later. In this report we describe the case in detail and discuss upon the fact, whether diagnostic laparoscopy is mandatory in such cases.

Keywords: Pneumatosis intestinalis, free abdominal air, norovirus, stem cell transplantation, acute myeloic leukemia, portal venous gas
\end{abstract}

Volume 9 Issue 2 - 2018

\author{
Stefan Reischl, Ihsan Ekin Demir, Philipp- \\ Alexander Neumann, Helmut Friess, Dirk \\ Wilhelm \\ Department of Surgery, Technical University of Munich, School \\ of Medicine, Klinikum rechts der Isar, Germany
}

Correspondence: Stefan Reischl, Department of Surgery, Technical University of Munich, School of Medicine, Klinikum rechts der Isar, Munich, Germany, Email stefan.reischl@tum.de

Received: February 12, 2018 | Published: April 16, 2018

\section{Introduction}

Pneumatosis intestinalis (PI) is a pathological condition defined as collection of gas within the intestinal wall, typically diagnosed in CT scans. Usually the gas is located to the colon (46\%), the small bowel $(27 \%)$, the stomach $(5 \%)$ or in combined patterns $(22 \%)$. ${ }^{1}$ In the most severe cases PI is caused by potentially life-threatening intestinal ischemia, which indicates the need for immediate surgical treatment. But in fact there is a broad variety of benign to severe causes for PI ranging from obstruction, cancer, volvulus, ulcer, hernia, trauma to inflammatory bowel diseases, diverticulitis, iatrogenic causes and viral infection., ${ }^{2,3}$ We present herein the case of a 19 yearold male patient, who developed PI following hematopoietic stem cell transplantation (HSCT) after acute myeloic leukemia (AML) with concomitant norovirus infection.

\section{Case report}

The 19 year-old male patient entered the hospital through the emergency department with a cough, fever of $38.9^{\circ} \mathrm{C}$ and diarrhea of 3-4 times per day. He suffered from AML type FAB M6 which had first been diagnosed 9 months before. Following diagnosis the AML has been treated by induction chemotherapy (cytarabine and daunorubicin, 7+3 regimen) and 2 cycles of consolidation chemotherapy with cytarabine followed by haploidentical stem cell transplantation donated by his mother 4 months ago. The respiratory symptoms caused by an acute graft-versus-host disease (GvHD) of the lung improved rapidly under intensified immunosuppressive therapy with prednisolone and mycophenolic acid.

However, during the further course he developed diarrhea, which got worse with defecations up to 20 times per day and the need for intravenous potassium substitution. The virological testing of the stools revealed norovirus infection, while the tests for adeno- and rotavirus and bacterial infections were negative. A GvHD of the colon was excluded by colonoscopy, as a biopsy of the mucosa revealed signs of acute infection without clear stigmata of GvHD. Under reduction of the immunosuppressive medication the stool frequency decreased to 4 times per day and the patient could finally be dismissed from the hospital. To this time, no specific therapy deemed necessary.

A few days later he was readmitted to the hospital for fever of $38.6^{\circ} \mathrm{C}$ and a cough. A CT scan of the chest showed symmetrical inflammatory infiltrates of the lungs. Additionally a massive PI and pneumoperitoneum was detected in the lower sections. The subsequent CT scan of the abdomen revealed pneumatosis mainly localized to the colon and also free abdominal air, while portal venous gas (PVG) could be excluded (Figure 1). Under immunosuppression the leucocyte count was $1.4 \mathrm{G} / 1$ and thus not usable for detection of inflammation or infection. The CRP $(2.0 \mathrm{mg} / \mathrm{dl})$ and lactate levels $(2.2 \mathrm{mmol} / \mathrm{l})$ were only slightly elevated. Clinically, the patient had an almost asymptomatic abdomen with only a slight tenderness but no abdominal guarding and lively bowel sounds. At first an interdisciplinary decision to perform a conservative treatment approach was made by the surgical and gastroenterological consultant, due to the benign symptomatology and the abdominal conditions. Antibiotic (meropenem and metronidazole), antiviral (ganciclovir) and supportive therapy (high-calorie diet) was conducted. Although infection signs continuously decreased under this treatment and the patient remained stable, further discussions ended up with indication for exploratory laparoscopy, as due to the immunosuppression of the patient, clinical signs could be misleading. After informed consent and preoperative preparation, the patient was brought to the operating room and diagnostic laparoscopy was performed (Figure 2).

During laparoscopy extensive pneumatosis of the complete colon with focus to the transverse colon became apparent. The small intestine was not affected by pneumatosis. Particular attention was spent to the appendix, which was also without pathological findings. The liver and the gallbladder were unremarkable. Fortunately, there were no signs of perforation, ischemia or inflammation, so no further intervention had to be performed. After taking a smear of the serous ascites a drainage tube was inserted and the operation finished. The patient 
recovered well from the operation and after assessing the ascites sterile by microbiologic examination, the drainage was removed on postoperative day 4 . With recurrent diarrhea and improved respiratory problems the patient was dismissed from the hospital 5 days later.
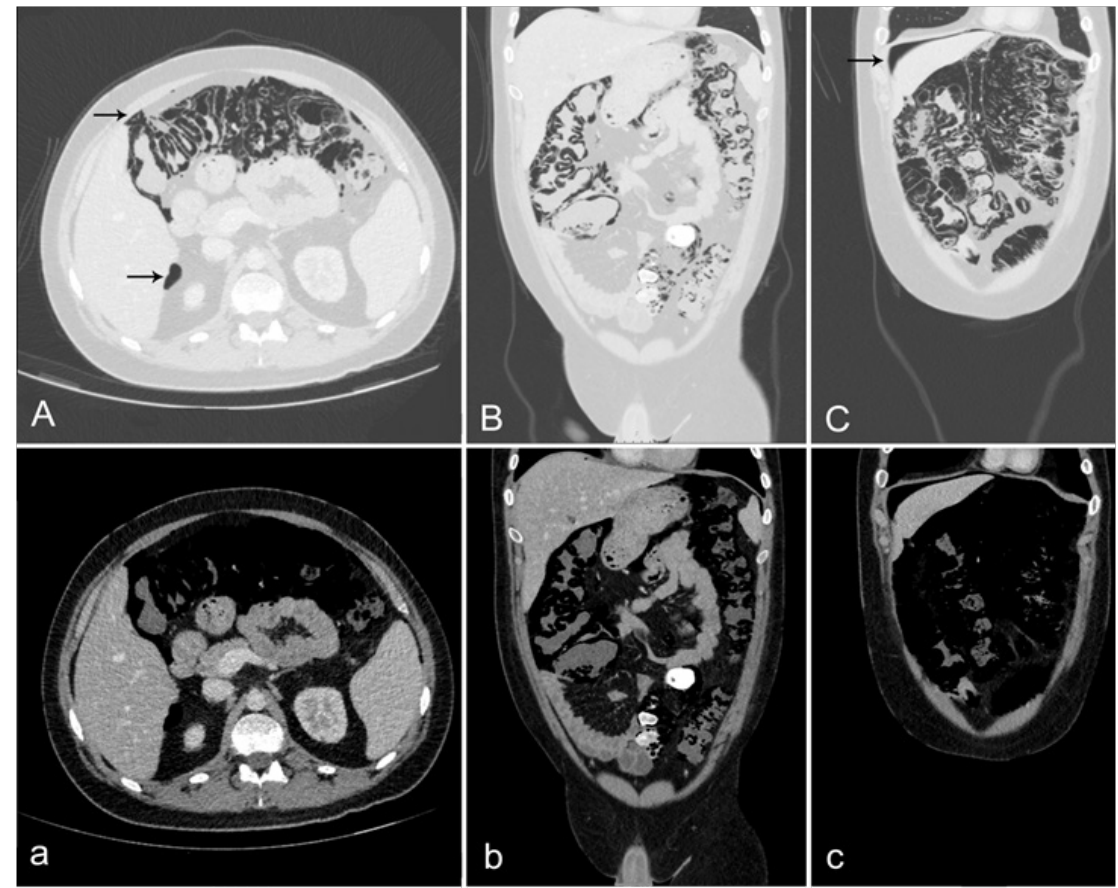

Figure I CT Scan of the abdomen was performed after PI was diagnosed incidentally. Contrasted with iodine, portalvenous phase. Axial (A) and coronary (B, C) sections of the abdomen. Sections are displayed in lung (Capital letters) and soft tissue window (small letters). Massive PI focused to the colon was visible. B) No portalvenous gas was observed (A, B). Scans showed perihepatic free abdominal air. (A,C) (Black arrows). No ascites could be detected.

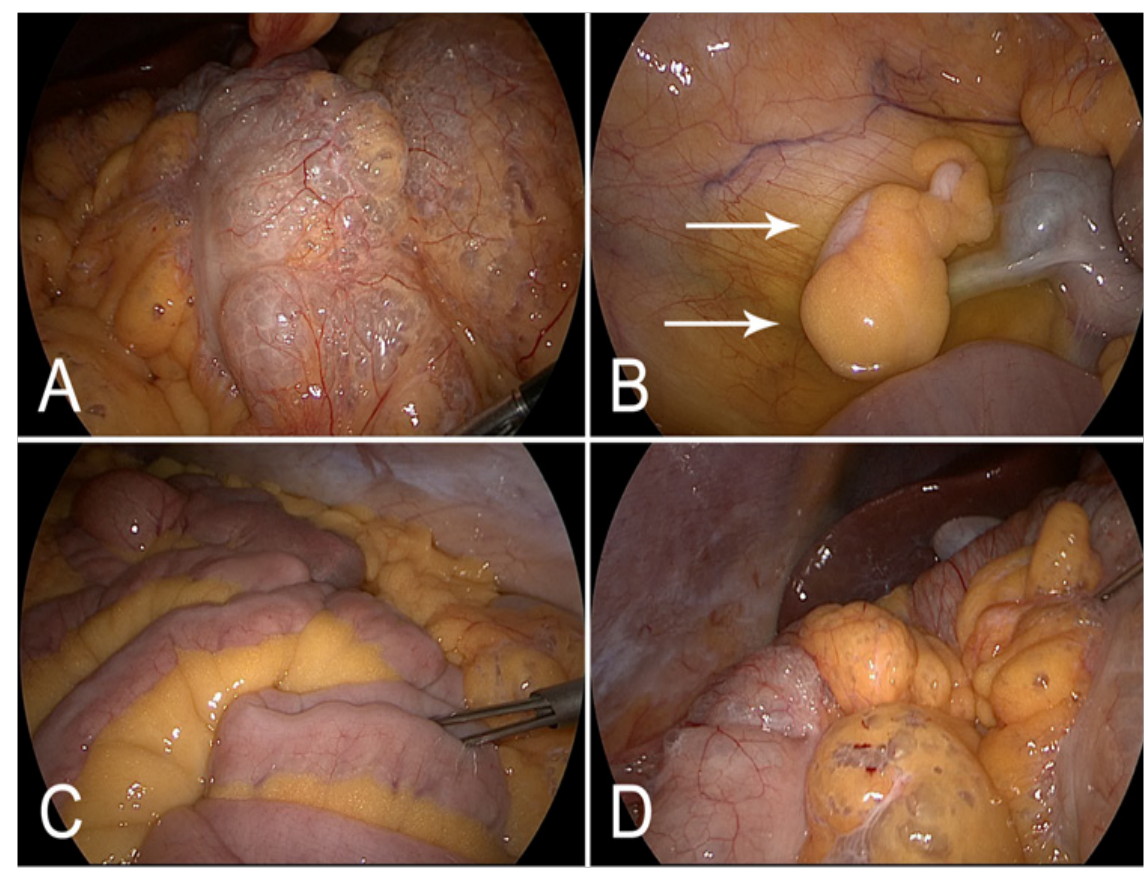

Figure 2 Diagnostic laparoscopy was performed after detection of $\mathrm{PI}$ and free abdominal air in the CT scan. (A) Massive pneumatosis predominantly of the transverse colon. B) The appendix (arrows) was inconspicuous. Serous ascites was present. (C) The small intestine was not affected by Pneumatosis. D) Pneumatosis also affected abdominal fat tissue. Liver and gallbladder were without pathological findings. 
Table I Causes for intestinal pneumatosis. $\mathrm{N}$ number of patients with intestinal pneumatosis. RATIO percentage of patients with this pathology. MORTALITY number of patients dying from pneumatosis in this group. MORTALITY RATE mortality rate of patients in this group Table adapted from ${ }^{2}$

\begin{tabular}{|c|c|c|c|c|}
\hline $\begin{array}{l}\text { Causes for } \\
\text { pneumatosis }\end{array}$ & $\mathbf{N}$ & Ratio (\%) & Mortality & $\begin{array}{l}\text { Mortality } \\
\text { rate (\%) }\end{array}$ \\
\hline Mechanical & 36 & 47,3 & 7 & 19,4 \\
\hline \multicolumn{5}{|c|}{ (Obstruction, cancer, volvolus, hernia) } \\
\hline Vascular & 35 & 46,1 & II & $3 I, 4$ \\
\hline \multicolumn{5}{|c|}{ (Intestinal infarction) } \\
\hline Inflammation & 5 & 6,6 & 0 & 0,0 \\
\hline \multicolumn{5}{|c|}{ (Crohn's disease, diverticulitis, ulceration) } \\
\hline Total & 76 & 100 & 18 & 23,7 \\
\hline
\end{tabular}

\section{Discussion}

The immediate cause for PI in this case is not clear, but presumably the prolonged course of the norovirus infection facilitated by immunosuppression might play an important role. While in immune-competent individuals norovirus infection only lasts a few days, it can cause a protracted diarrhea with prolonged viral shedding in feces in immunocompromised patients up to 1 year., ${ }^{4,5}$ A norovirus prevalence of $25 \%$ in transplanted patients (HSCT and solid organ transplantation) has been reported. ${ }^{6}$ The present patient had been suffering from diarrhea for 3 weeks until norovirus infection was finally diagnosed. In our case detection of PI was only an incidental finding on a CT scan of the thorax for pneumonia. Development of PI due to norovirus infection is a rare event. However, a retrospective study assumed a correlation between immunodeficiency with concomitant norovirus infection in the development of PI. The pediatric patient collective ( 0.2 to 14.8 years, median 1.4 years) comprised four patients with simultaneous existence of PI and norovirus infection under immunosuppression because of chemotherapy or HSCT. ${ }^{7}$ None of those four patients died. However, also severe courses of norovirus infections in adults with gastrointestinal necrosis have been reported. ${ }^{8-10}$

Obviously in the presented patient PI and pneumoperitoneum was caused by transmigration of gas through the bowel walls, as no perforation could be detected in diagnostic laparoscopy. In accordance, another patient with PI and concomitant free abdominal air due to AIDS was reported, who showed no perforation in exploratory laparotomy. ${ }^{11}$ The incidence of PI seems to correlate to immunosuppressive diseases in general, as a variety of cases of PI in immunocompromised patients by various reasons as transplantation, AIDS, chemotherapy or leukemia without proof of an infection have been reported. However, it remains unclear if additional infection is a necessary condition to cause PI or if immunosuppression itself can result in PI.

In our case pneumatosis was limited to the bowel walls without presence of air in the vascular system. However, prognosis is much worse, if PVG is detected with PI. Mortality rates of up to $30 \%$ have been reported, due to a higher rate of ischemic conditions than in exclusive PI. ${ }^{12,13}$ In patients with PVG and mesenteric ischemia mortality raises to $50 \% .^{13,14}$ However, prognosis of PVG is highly dependent of the underlying pathology. While evidence for ischemia indicates an immediate surgical approach, conservative management of PI and PVG can be a reasonable strategy if ischemia is unlikely.

\section{Conclusion}

From the surgical point PI is always an indicator for an acute intestinal complication, especially in presence of pneumoperitoneum, and in general exploration laparotomy or laparoscopy is performed. If the patient is doing well and does not show clinical signs of an acute abdomen, decision for operation can be difficult and should be made as an interdisciplinary approach. In this patient, the immunosuppression of the patient could have masked an abdominal infection, so finally laparoscopy was performed. As this was without evidence for intestinal perforation or ischemia, one might argue whether surgery was indicated or a conservative treatment should have been maintained. Maybe, the absence of portal vein air embolization, that is typical for intestinal ischemia, could have been used as another indicator for a benign cause of PI, however it does not exclude intestinal perforation. But one should also consider the circumstances for PI. As so, Norovirus infection is a known reason for pneumatosis intestinalis as is immunosuppression. Presumably, immunodeficient patients with PI and norovirus without pneumoperitoneum can be safely treated conservatively and monitored clinically. In case of pneumoperitoneum, however, we recommend exclusion of perforation with minimal trauma by diagnostic laparoscopy.

\section{Acknowledgements}

None.

\section{Conflict of interest}

The author declares no conflict of interest.

\section{References}

1. Morris MS, Gee AC, Cho SD, et al. Management and outcome of pneumatosis intestinalis. Am J Surg. 2008;95(5):679-82.

2. Lassandro F, Mangoni de Santo Stefano ML, Porto AM, et al. Intestinal pneumatosis in adults: diagnostic and prognostic value. Emerg Radiol. 2010;17(5):361-5

3. Gagliardi G, Thompson IW, Hershman MJ, et al. Pneumatosis coli: a proposed pathogenesis based on study of 25 cases and review of the literature. Int J Colorectal Dis. 1996;11(3):111-8.

4. Roddie C, Paul JP, Benjamin R, et al. Allogeneic hematopoietic stem cell transplantation and norovirus gastroenteritis: a previously unrecognized cause of morbidity. Clin Infect Dis. 2009;49(7):1061-8.

5. Westhoff TH, Vergoulidou M, Loddenkemper C, et al. Chronic norovirus infection in renal transplant recipients. Nephrol Dial Transplant. 2009;24(3):1051-3.

6. Ye X, Van JN1, Munoz FM, et al. Noroviruses as a Cause of Diarrhea in Immunocompromised Pediatric Hematopoietic Stem Cell and Solid Organ Transplant Recipients. Am J Transplant. 2015;15(7):1874-81.

7. Kim MJ, Kim YJ, Lee JH, et al. Norovirus: a possible cause of pneumatosis intestinalis. J Pediatr Gastroenterol Nutr. 2011;52(3):3148 .

8. Yasuda H, Okita Y, Imaoka H, et al. Intestinal necrosis due to norovirus enteritis. Clin J Gastroenterol. 2015;8(1):10-3. 
9. Pawa N, Vanezis AP, Tutton MG. Spontaneous bowel perforation due to norovirus: a case report. Cases J. 2009;2:9101.

10. Zenda T, Kaneko S, Noriki S. Norovirus gastroenteritis accompanied by ischemic colitis: a case report. Hiroshima J Med Sci. 2010;59(4):83-5.

11. Wood BJ, Kumar PN, Cooper C, et al. Pneumatosis intestinalis in adults with AIDS: clinical significance and imaging findings. AJR Am J Roentgenol. 1995;165(6):1387-90.

12. Faberman RS, Mayo-Smith WW. Outcome of 17 patients with portal venous gas detected by CT. AJR Am J Roentgenol. 1997;169(6):1535-8.
13. Schindera ST, Triller J, Vock P, et al. Detection of hepatic portal venous gas: its clinical impact and outcome. Emerg Radiol. 2006;12(4):164-70.

14. Wiesner W, Mortelé KJ, Glickman JN, et al. Pneumatosis intestinalis and portomesenteric venous gas in intestinal ischemia: correlation of CT findings with severity of ischemia and clinical outcome. AJR Am J Roentgenol. 2001;177(6):1319-23. 